\title{
Impact of Plasma Donepezil Concentration on Behavioral and Psychological Symptoms of Dementia in Patients with Alzheimer's Disease
}

\author{
Yoshiyuki Kagawa $^{a}$ Yoshiaki Yamamoto ${ }^{a}$ b Ayami Ueno $^{a}$ Kengo Inomata ${ }^{a}$ \\ Mayu Tezuka ${ }^{a}$ Takashi Osawa ${ }^{a}$ Yasuharu Yazawa ${ }^{a}$ Toshio Maeda ${ }^{a}$ \\ Tomokazu Obi ${ }^{\text {b }}$ \\ aSchool of Pharmaceutical Sciences, Laboratory of Clinical Pharmaceutics, University of Shizuoka, Shizuoka, Japan; \\ ${ }^{b}$ Department of Clinical Research, National Epilepsy Center, Shizuoka Institute of Epilepsy and Neurological \\ Disorders, Shizuoka, Japan
}

\section{Keywords}

Alzheimer's disease $\cdot$ Behavioral and psychological symptoms of dementia - Donepezil · Pharmacokinetics .

Polymorphism

\begin{abstract}
Background/Aims: The behavioral and psychological symptoms of dementia (BPSD) detract from the quality of life of not only dementia patients but also their family members and caregivers. Donepezil is used to treat Alzheimer's disease and is metabolized via cytochrome P450 (CYP) 2D6 and CYP3A4/5. It is controversial whether donepezil improves or exacerbates BPSD. This study investigated the relationships among BPSD, the pharmacokinetics of donepezil including its metabolite, 6-O-desmethyl donepezil, genetic polymorphisms of CYPs and P-glycoprotein, and patient backgrounds in 52 patients with Alzheimer's disease. Methods: BPSD were assessed using the Neuropsychiatric Inventory (NPI), with scores $\geq 20$ points defined as severe BPSD. Plasma donepezil and 6-O-desmethyl donepezil concentrations were measured using liquid chromatography-tandem mass spectrometry. Results: Although significant relationships between NPI scores and plasma donepezil concentrations were not seen, none of the 15 patients (29\%) with high plasma
\end{abstract}

karger@karger.com www.karger.com/dee

Karger $\stackrel{\text { ' }}{5}$

BOPEN ACCESS
(C) 2021 The Author(s)

Published by S. Karger AG, Basel

This is an Open Access article licensed under the Creative Commons Attribution-NonCommercial-4.0 International License (CC BY-NC) (http://www.karger.com/Services/OpenAccessLicense), applicable to the online version of the article only. Usage and distribution for commercial purposes requires written permission. donepezil concentrations ( $\geq 60 \mathrm{ng} / \mathrm{mL}$ ) developed severe BPSD. Polymorphisms of CYP2D6, CYP3A5, and $A B C B 1$ did not influence NPI scores. There were no significant relationships between NPI and patient background factors such as dosing regimen, concomitant use of other drugs, or laboratory test results. Two patients who underwent multiple blood samplings over 2 years showed an inverse correlation between plasma donepezil concentrations and NPI scores. Discussion/Conclusions: These results indicate that higher plasma concentrations of donepezil contribute to preventing or alleviating rather than developing or deteriorating BPSD.

\section{(c) 2021 The Author(s).}

Published by S. Karger AG, Basel

\section{Introduction}

Alzheimer's disease (AD) is the most common type of dementia, representing $50-70 \%$ of dementia cases [1]. Dementia, including $\mathrm{AD}$, encompasses both cognitive and noncognitive symptoms, referred to as the behavioral and psychological symptoms of dementia (BPSD). BPSD include agitation, aggression, apathy, wandering, and sleep disturbance [2]. Exacerbation of BPSD increases the burden of caregivers, in addition to reducing the quality of life of patients [3,4]. Although BPSD were pre- 
viously treated using typical or atypical antipsychotics, it became clear that their efficacy was limited and that these drugs had marked adverse effects. Moreover, in 2005, the US Food and Drug Administration issued an advisory that atypical antipsychotics increase the risk of stroke when used to treat BPSD in elderly patients with dementia [5]. Some physicians in Japan use Japanese Kampo medicines, notably Yokukansan, for the treatment of BPSD [6], and there are reports that Yokukansan is effective for decreasing BPSD symptoms [7, 8]. The etiopathogenesis of BPSD is complicated and multifactorial, encompassing brain lesions and types of dementia, changes in neurotransmission and neuromodulation, physical disorders and pain, psychological and environmental perspective, personality traits, and life events [9]. Antidementia drugs, such as donepezil, are used not only for the improvement of cognitive disorders but also for the alleviation of BPSD. The effects of antidementia medicines on BPSD have, however, been controversial. Some reports showed improvements in BPSD by donepezil [10$14]$, while others had contrasting outcomes $[15,16]$.

Donepezil is metabolized by cytochrome p-450 (CYP) isoenzymes in humans, primarily by CYP2D6 and to a lesser extent by CYP3A4/5 [17]. A major metabolite of donepezil is 6-O-desmethyldonepezil (6ODD), produced by CYP2D6. 6ODD is an active metabolite with potency to inhibit acetylcholinesterase comparable to that of donepezil [18]. However, based on animal studies, the transfer of 6ODD into the brain seems to be low [19]. The clinical contribution of 6ODD to the efficacy of donepezil treatment, therefore, remains unclear. The phenotypes of CYP2D6 polymorphisms are divided into four groups (ultrarapid metabolizer, extensive metabolizer, intermediate metabolizer, and poor metabolizer), according to their enzymatic activity [20]. It was reported that a higher frequency of the CYP $2 D 6^{*} 10$ allele, which encodes a low-activity form of the enzyme, was seen in responder Alzheimer's patients than in nonresponders [21]. This result suggests that higher blood concentrations of donepezil contribute to improved efficacy of the drug. Since the $6 \mathrm{ODD} /$ donepezil concentration ratio in blood correlates with the enzymatic activity of CYP2D6 in individual patients, the ratio shows CYP2D6 phenotype dependency [22]. The efflux of donepezil from the brain to peripheral blood is achieved via P-glycoprotein encoded by the $A B C B 1$ gene [23]. Patients with the T/T/T haplotype of three major $A B C B 1$ polymorphisms may show lower plasma donepezil concentrations and better clinical outcomes than those with other genotypes, but the differences are not significant [20]. There have been no reports on whether the genetic polymorphisms of CYP2D6, $C Y P 3 A 5$, and $A B C B 1$ influence the development of BPSD in patients receiving donepezil.

We recently reported the impact of polymorphisms of metabolic enzymes and transporters of donepezil on plasma donepezil pharmacokinetics in $\mathrm{AD}$ [22]. In this study, we investigated the influence of plasma concentrations of donepezil and 6ODD, polymorphisms of CYP2D6, $C Y P 3 A 5$, and $A B C B 1$, and patient backgrounds on the development of BPSD.

\section{Materials and Methods}

\section{Chemicals}

Donepezil, 6ODD, and escitalopram were obtained from Tokyo Chemical Industry (Tokyo, Japan), Toronto Research Chemicals Inc. (North York, Canada), and R\&D Systems Inc. (Minneapolis, MN, USA), respectively. All other chemicals were commercially available and of analytical grade.

\section{Patient Selection}

Fifty-two blood samples from AD patients were used. All patients received treatment with donepezil at the hospital of the Shizuoka Institute of Epilepsy and Neurological Disorders between January 2014 and July 2020. The backgrounds of the enrolled patients are shown in Table 1.

\section{Drug Administration and Blood Sampling}

Donepezil (3-10 mg/day) was administered once daily for at least 2 months before blood sampling. Blood samples were obtained on an average of $2.16 \mathrm{~h}$ (range $0.92-4.50$ ) after oral administration (Table 1).

\section{Pharmacogenetic Analysis}

Genomic DNA of patients was extracted from whole blood using a MagNA Pure Compact ${ }^{\circledR}$ (Roche Molecular Diagnostics, Pleasanton, CA, USA). Polymorphisms of CYP2D6 * $1,{ }^{*} 2, * 4, * 5$, $* 10$, and $* 2$ A were detected by the Luminex ${ }^{\circledR} 100 / 200{ }^{\mathrm{TM}} \mathrm{xMAP}^{\circledR}$ platform using the $x$ TAG ${ }^{\circledR} C Y P 2 D 6$ RUO kit v3 (Luminex, Austin, TX, USA). Ultrarapid metabolizer, extensive metabolizer, intermediate metabolizer, and poor metabolizer were defined as described previously [22]. Polymorphism of CYP3A $5^{*} 3$ was detected based on the PCR-RFLP method reported by Balram et al. [24] and slightly modified by our laboratory. Polymorphisms of $A B C B 1$ G2677T/A and C3435T were also detected using the PCR-RFLP method reported by Tanabe et al. [25] and slightly modified by ourselves.

\section{Evaluation of BPSD and Cognitive Impairment}

The Neuropsychiatric Inventory (NPI), consisting of 10 items (total score: 120 points), was adopted as the instrument for evaluating symptoms of BPSD [26]. The NPI score was used to divide patients into two groups: high BPSD with NPI $\geq 20$ and low BPSD with NPI $<20$ [27]. The Mini-Mental State Examination (MMSE) was implemented to assess the degrees of functional and cognitive impairment [28]. 
Table 1. Patient backgrounds

\begin{tabular}{|c|c|c|c|c|c|c|c|}
\hline \multicolumn{2}{|l|}{ Sex } & 52 & & \multicolumn{4}{|c|}{ Male 20; female 32} \\
\hline \multicolumn{2}{|c|}{ Albumin, $\mathrm{g} / \mathrm{dL}$} & 27 & 4.03 & 0.37 & \multicolumn{3}{|l|}{$3.1-4.5$} \\
\hline \multicolumn{2}{|c|}{ AST, IU/L } & 52 & 23.7 & 7.55 & \multicolumn{3}{|l|}{$13-51$} \\
\hline \multicolumn{2}{|l|}{ ALT, IU/L } & 52 & 17.0 & 7.79 & \multicolumn{3}{|l|}{$7-52$} \\
\hline \multicolumn{2}{|c|}{ Body weight, $\mathrm{kg}$} & 52 & 50.8 & 10.6 & \multicolumn{3}{|l|}{$32.5-91.0$} \\
\hline \multicolumn{2}{|c|}{ MMSE } & 25 & 19.0 & 6.3 & \multicolumn{3}{|l|}{$3-28$} \\
\hline \multicolumn{2}{|c|}{ Donepezil dose, mg/kg } & 52 & 0.124 & 0.0534 & \multicolumn{3}{|l|}{$0.0549-0.270$} \\
\hline \multicolumn{2}{|c|}{ Blood sampling time after administration } & 52 & 2.16 & 0.834 & \multicolumn{3}{|l|}{$0.92-4.50$} \\
\hline \multicolumn{2}{|c|}{ Concomitant use of psychotropics } & 7 & \multicolumn{5}{|c|}{ Tiapride 5 , sertraline 1 , and flunitrazepam 1} \\
\hline Patient 1 & 5 & Male & 64 & 52 & $5-8$ & EM & $* 3 / * 3$ \\
\hline Patient 2 & 6 & Male & 76 & 43 & 5 & EM & $* 3 / * 3$ \\
\hline
\end{tabular}

SD, standard deviation; AST, aspartate aminotransferase; ALT, alanine aminotransferase; $\gamma$-GTP, $\gamma$-glutamyl transpeptidase; MMSE, MiniMental State Examination.

Pharmacokinetic Analysis

Plasma donepezil and 6ODD concentrations were determined by high-performance liquid chromatography-tandem mass spectrometry, using the modified method of Pilli et al. [29] and Shah et al. [30]. Escitalopram was used as an internal standard. The minimum detection limit of both donepezil and 6ODD was $0.10 \mathrm{ng} / \mathrm{mL}$. To compare individual pharmacokinetic data, we calculated the concentration/dose $(\mathrm{C} / \mathrm{D})$ ratio of donepezil or 6ODD as the plasma concentration $(\mathrm{ng} / \mathrm{mL})$ divided by the weight-adjusted dose $(\mathrm{mg} / \mathrm{kg})$.

\section{Statistical Analysis}

Statistical analyses were conducted using SPSS 23.0 (SPSS, Inc., Chicago, IL, USA). Linear regression analysis was performed using Pearson's product-moment correlation coefficient. The $t$-test and Mann-Whitney U-test were used when comparing data of two independent groups with normal and non-normal distributions, respectively. The Tukey's HSD test was applied when comparing data of three or more groups. $p<0.05$ was considered to indicate a significant difference.

\section{Results}

\section{Influence of Patient Background Factors and}

Concomitant Drugs on BPSD

The NPI scores as an index of the development of BPSD did not correlate with the MMSE scores, patient age, body weight, or sex (Fig. 1). We did not find any significant relationship between NPI scores and serum albumin, alanine aminotransferase, aspartate aminotransferase, $\gamma$-glutamyl transpeptidase, total bilirubin, or serum creatinine (online suppl. Fig. 1; for all online suppl. material, see www.karger.com/doi/10.1159/000516938). NPI scores did not differ between patients receiving or not receiving psychotropics, memantine, or Yokukansan (online suppl. Fig. 2).

\section{Influence of the Plasma Concentration of Donepezil and Protein Polymorphisms on BPSD}

Figure 2 shows the relationships between NPI scores, plasma donepezil (total and free), 6ODD concentrations, and the $6 \mathrm{ODD} /$ donepezil concentration ratio. Interestingly, patients with high plasma concentrations of donepezil $(>60 \mathrm{ng} / \mathrm{mL})$ or 6 ODD $(>0.4 \mathrm{ng} / \mathrm{mL})$ did not develop severe BPSD, that is, their NPI scores did not reach or exceed 20 points. The donepezil dose (mg/day and $\mathrm{mg} /$ $\mathrm{kg}$ ) and $\mathrm{C} / \mathrm{D}$ ratios of donepezil and 6ODD did not directly correlate with NPI scores (online suppl. Fig. 3). However, patients with higher donepezil doses $(>0.15$ $\mathrm{mg} / \mathrm{kg}$ ) did not develop severe BPSD, reflecting the results of donepezil pharmacokinetics (online suppl. Fig. 3). Similarly, patients with higher donepezil C/D ratios that 
Fig. 1. Relationship between NPI scores and MMSE (a), age (b), body weight (c), and sex (d) in patients with Alzheimer's disease. The box plot presents the distribution of data based on the five-number summary. From the bottom: minimum, first quartile, median, third quartile, and maximum. NPI, Neuropsychiatric Inventory; MMSE, Mini-Mental State Examination.

Fig. 2. Relationship between NPI scores and plasma total (a) and free (b) concentrations of donepezil, plasma total 6ODD concentrations (c), and the 6ODD/donepezil concentration ratio (d) in patients with $\mathrm{AD}$. The plasma $6 \mathrm{ODD} /$ donepezil concentration ratio (metabolic ratio) was calculated by dividing the plasma 6ODD concentration by the plasma donepezil concentration. NPI, Neuropsychiatric Inventory; 6ODD, 6-O-desmethyl donepezil; $\mathrm{AD}$, Alzheimer's disease.

Donepezil Pharmacokinetics and BPSD
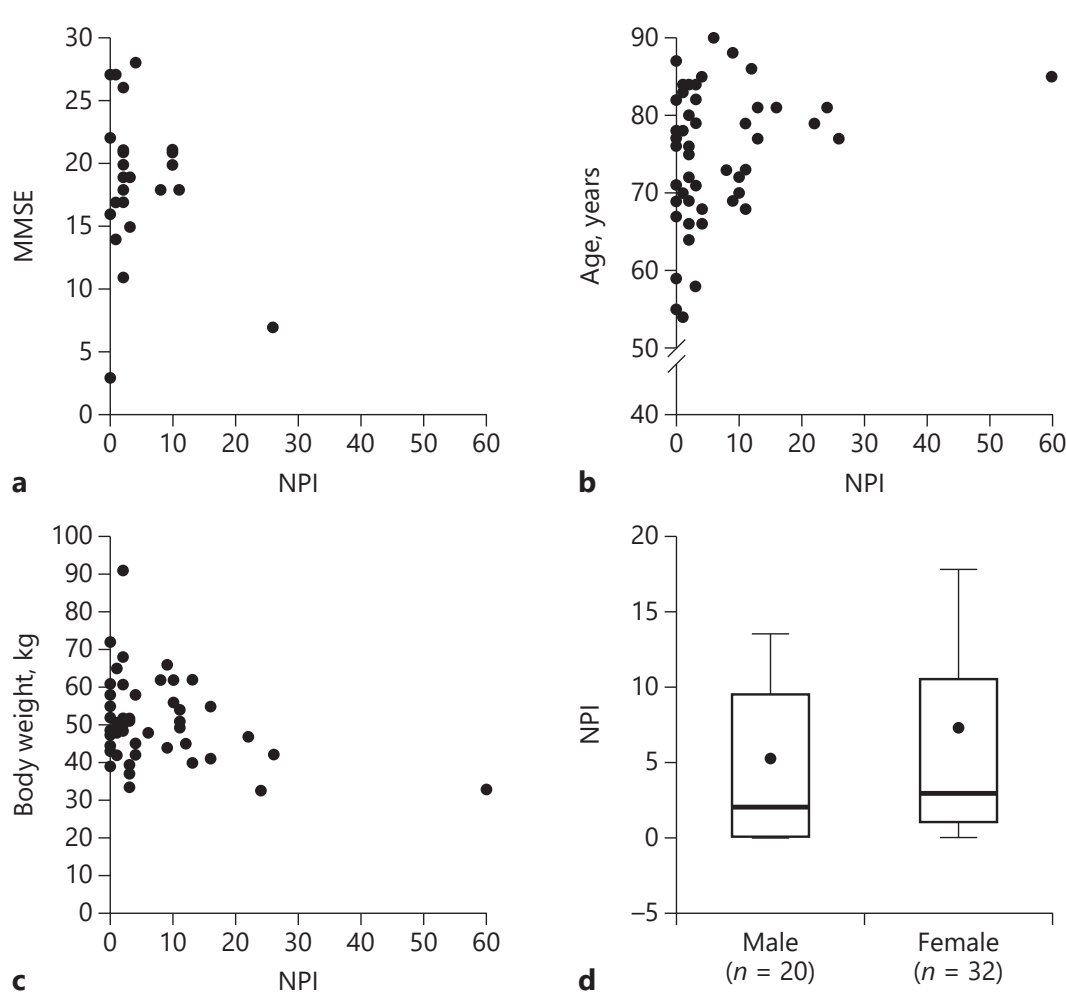

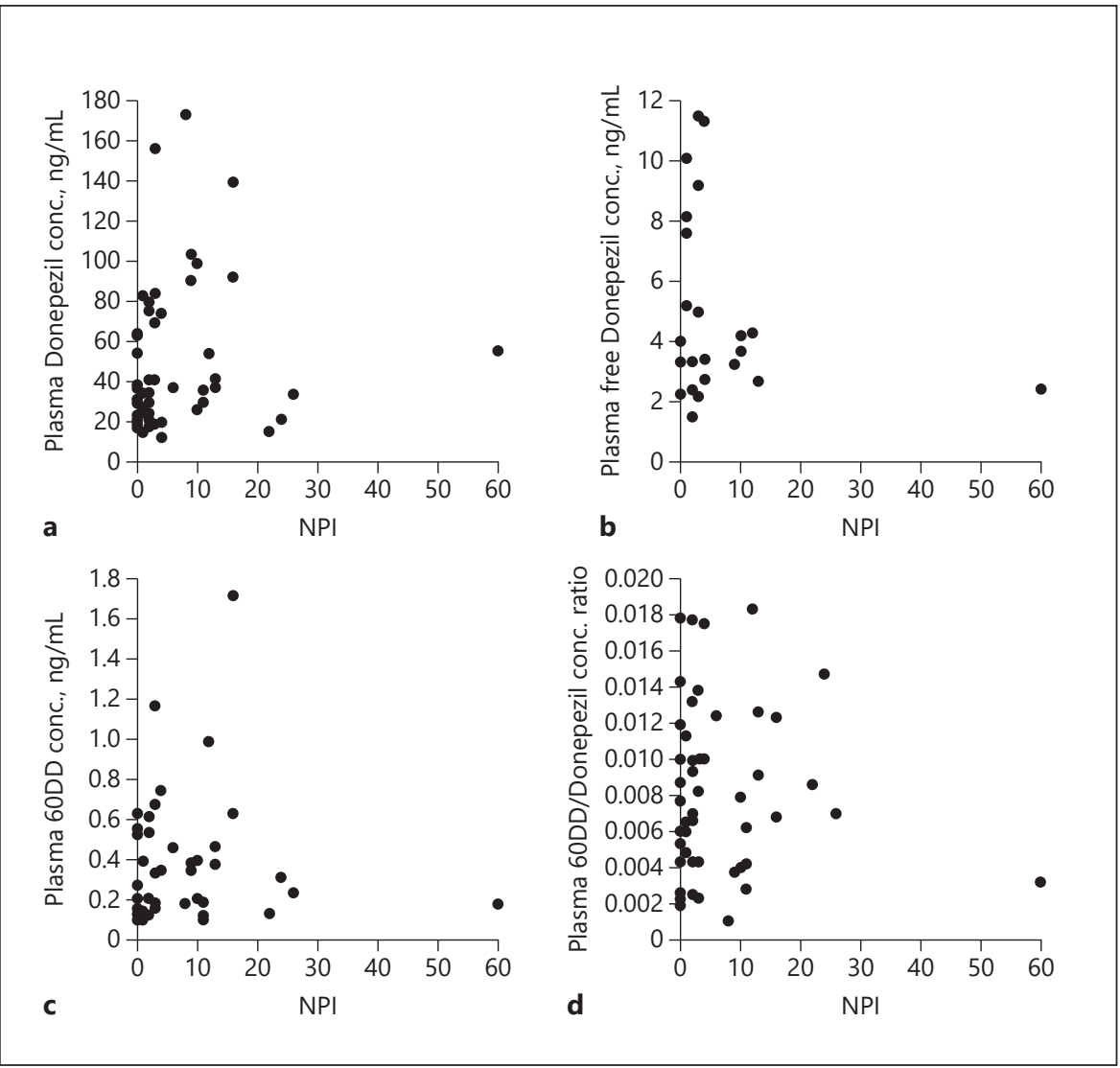


Fig. 3. Comparison of plasma donepezil (a), 6ODD (b) concentrations, 6ODD/donepezil concentration ratio (c), and donepezil $\mathrm{C} / \mathrm{D}$ ratio $(\mathbf{d})$ between patients with NPI $<$ or $\geq 20$ points. The box plot presents the distribution of data based on the fivenumber summary. From the bottom: minimum, first quartile, median, third quartile, and maximum. The concentration/dose (C/D) ratios of donepezil and 6ODD were calculated by dividing the plasma concentrations $(\mathrm{ng} / \mathrm{mL})$ by the weight-adjusted doses (mg/kg). The Mann-Whitney U-test was used to compare data of two independent groups. NPI, Neuropsychiatric Inventory; 60DD, 6-O-desmethyl donepezil.
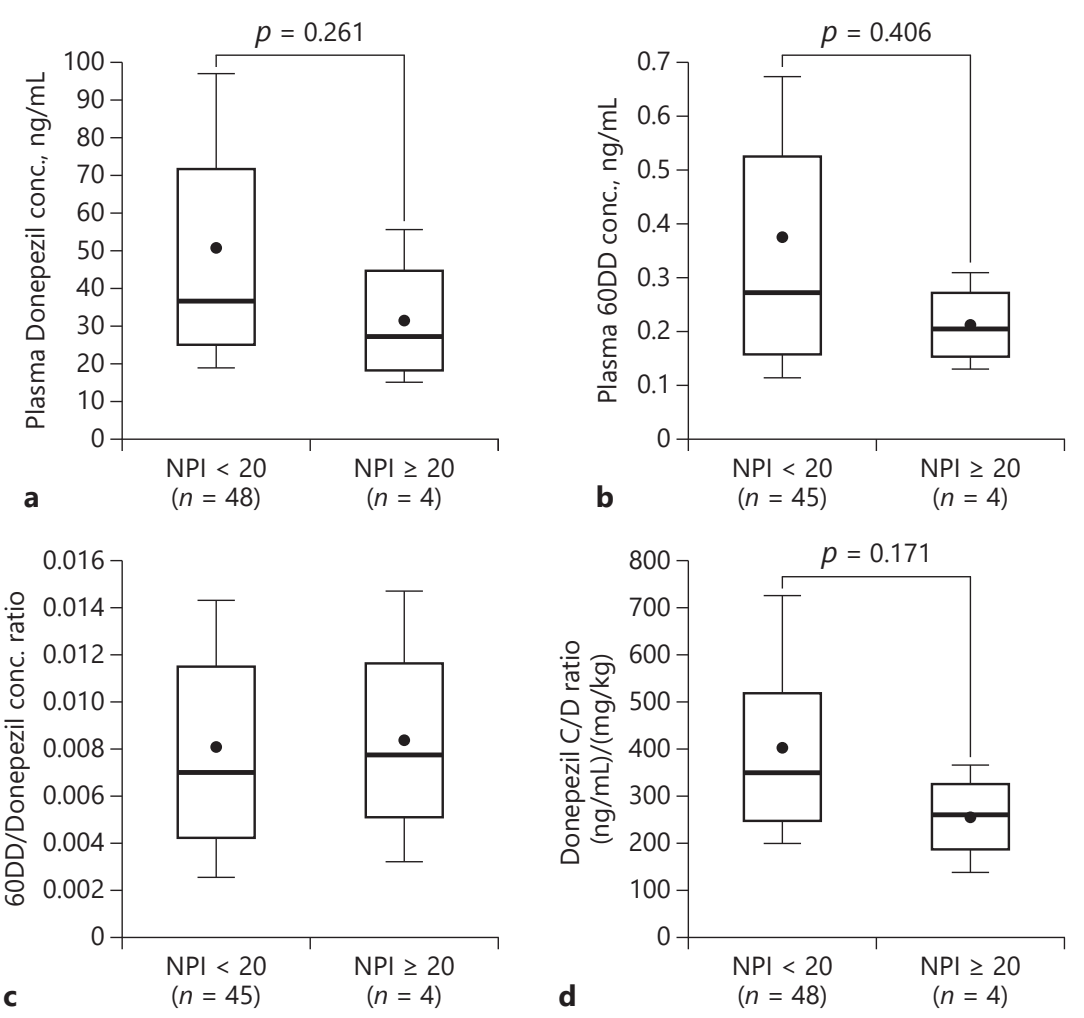

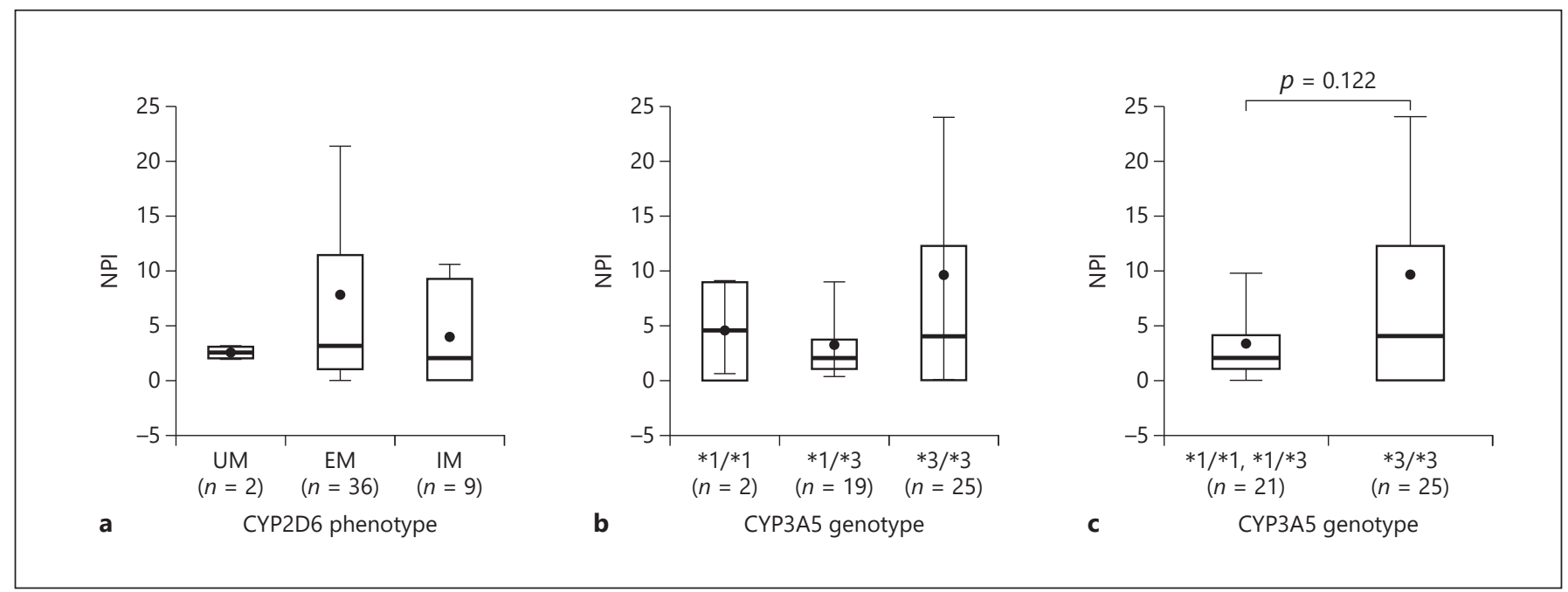

Fig. 4. Influence of CYP2D6 phenotypes (a), and CYP3A5 genotypes (b) and phenotypes (c) on NPI scores in patients with AD. Patients were classified into three groups according to the CYP2D6 genotype UM, EM, and IM. Patients were also divided into three or two groups according to the genotypes of CYP3A5. The box plot presents the distribution of data based on the five-number sum- mary. From the bottom: minimum, first quartile, median, third quartile, and maximum. The Mann-Whitney U-test was used to compare data of 2 independent groups. NPI, Neuropsychiatric Inventory; $\mathrm{AD}$, Alzheimer's disease; UM, ultrarapid metabolizer; EM, extensive metabolizer; IM, intermediate metabolizer. 
Fig. 5. Influence of $A B C B 1 G 2677 T / A$ (a), $C 3435 T$ (b) genotypes and the presence of $\mathrm{T}$ alleles $(\mathbf{c}, \mathbf{d})$ on NPI scores in patients with $\mathrm{AD}$. Patients were divided into three or two groups according to the $A B C B 1$ G2677T/A and C3435T genotypes or the presence of $\mathrm{T}$ alleles. The box plot presents the distribution of data based on the fivenumber summary. From the bottom: minimum, first quartile, median, third quartile, and maximum. NPI, Neuropsychiatric Inventory; $\mathrm{AD}$, Alzheimer's disease.

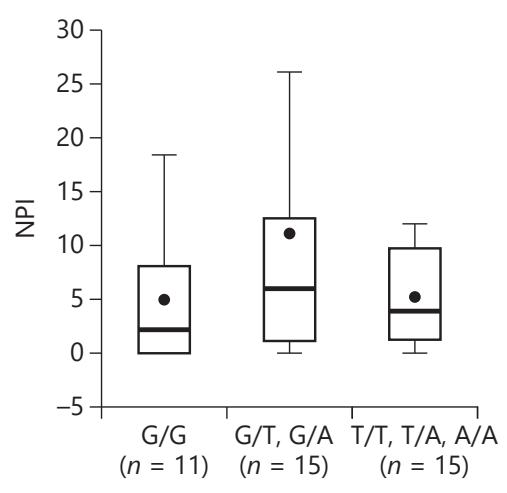

a

ABCB1 G2677T/A genotype

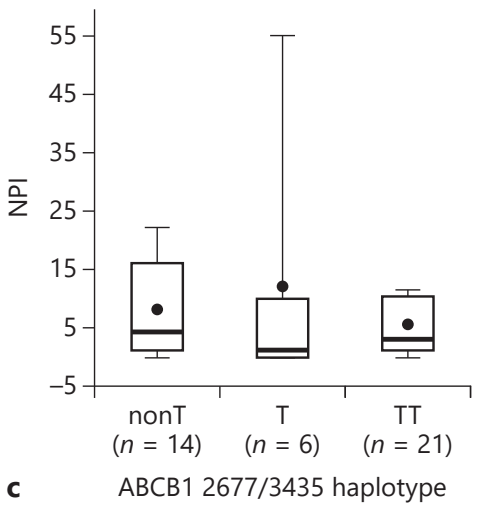

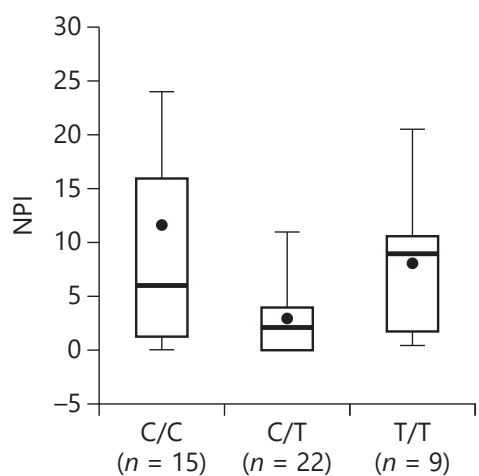

b

ABCB1 C3435T genotype

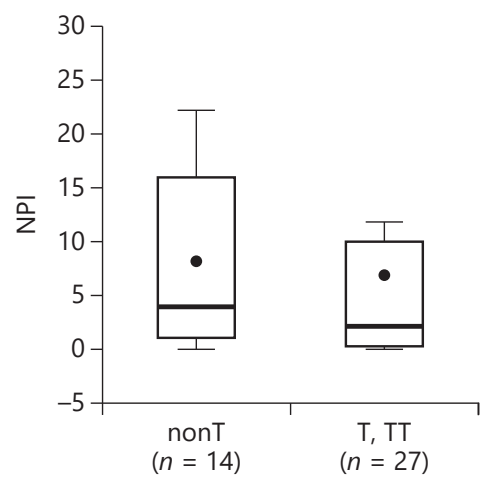

d $\quad A B C B 12677 / 3435$ haplotype represented total clearance of donepezil did not develop severe BPSD. We compared plasma concentrations of donepezil and 6ODD, plasma 6ODD/donepezil concentration ratios, and donepezil $\mathrm{C} / \mathrm{D}$ ratios between patients whose NPI scores were higher or lower than 20 points (Fig. 3). There were no significant differences between them, probably because of the small number of patients who developed severe BPSD. Figure 4 shows the influence of polymorphisms of CYP2D6 and CYP3A5 on NPI scores. There were no gene or phenotype impacts on NPI scores. Genotypes or haplotypes of ABCB1 2677 and 3435 also did not influence NPI scores (Fig. 5).

\section{Two Case Reports of Patients with Multiple Blood Samplings}

Five or six blood samplings were obtained over 2 years from two patients whose CYP2D6 phenotype was EM. Figure 6 shows the changes in their NPI scores. Remarkably, the changes in both patients were inversely associated with plasma donepezil concentration. The effect of concomitant use of memantine on BPSD was inconsistent between the two patients.

Donepezil Pharmacokinetics and BPSD

\section{Discussion}

As BPSD markedly lowers the quality of life of patients with dementia, their families, and caregivers, clinically effective treatments are needed for BPSD. The effects of donepezil on BPSD have been controversial [10-16]. To the best of our knowledge, this is the first attempt to clarify the relationships between the blood concentration of donepezil, 6ODD, and BPSD in patients with AD. Although this study did not find a linear relationship between plasma donepezil disposition and the development of BPSD, no patients whose plasma concentrations of donepezil exceeded $60 \mathrm{ng} / \mathrm{mL}$ developed severe BPSD. Moreover, the plasma donepezil concentrations in two patients with multiple blood samplings over 2 years showed an inverse relationship to the changes in NPI scores. Patients with high plasma donepezil concentrations did not develop any noticeable side effects. These results indicate that if a patient develops severe BPSD and the plasma donepezil concentration near the peak is $<60$ $\mathrm{ng} / \mathrm{mL}$, an increased dose to raise the concentration may contribute to improving severe BPSD. The optimal thera- 


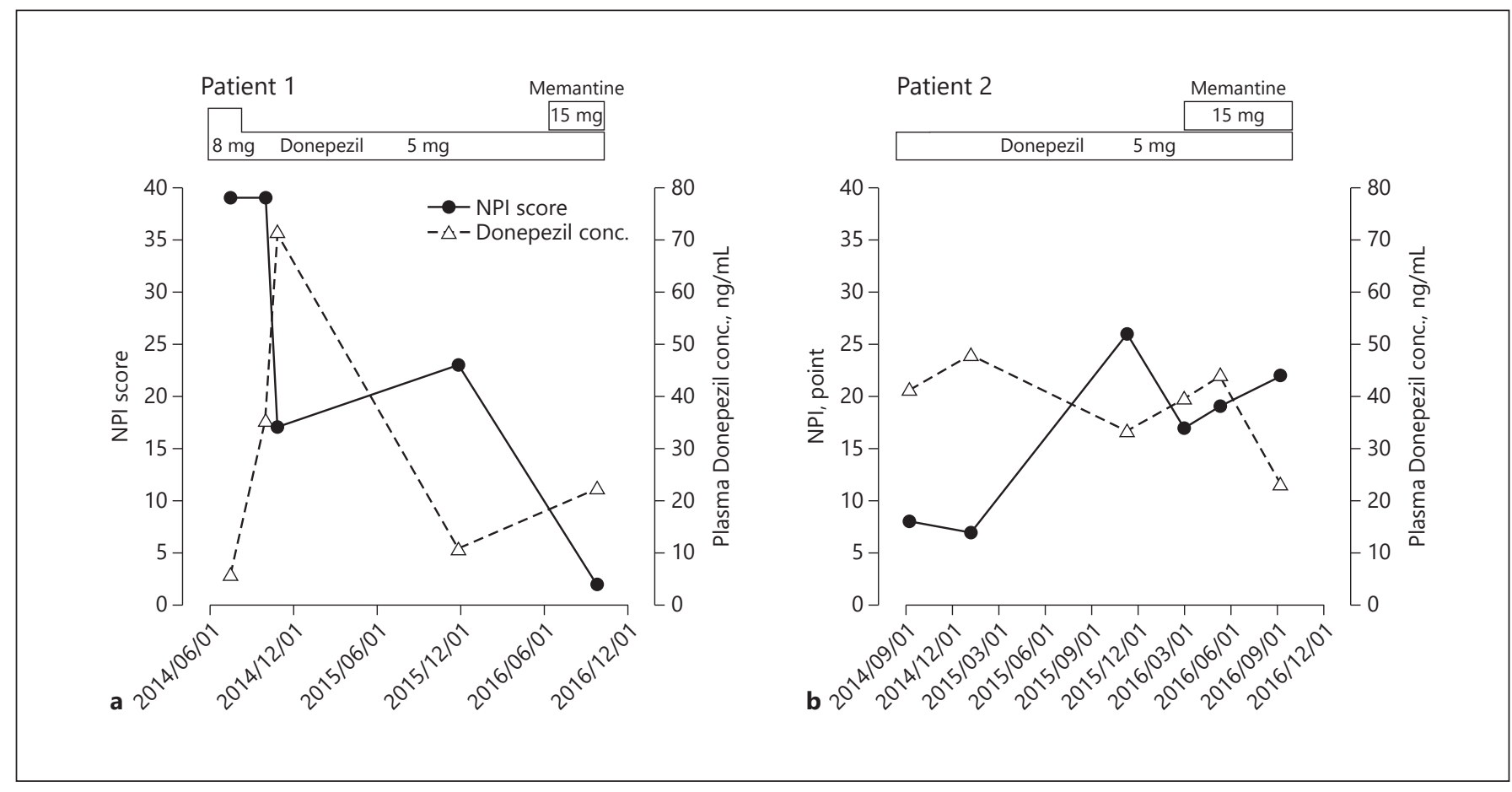

Fig. 6. Changes in NPIscores and plasma donepezil concentrations in two patients with multiple blood samplings. Two patients (patient 1, a; patient 2, b) underwent five or six blood samplings over 2 years to determine plasma donepezil concentrations and NPI scores. An inverse correlation between plasma donepezil concentrations and NPI scores was observed. NPI, Neuropsychiatric Inventory.

peutic range of donepezil is reported to be $50-75 \mathrm{ng} / \mathrm{mL}$ at the trough in plasma [31]. Noetzli et al. [32] reported that a one-compartment model describes the plasma disposition of donepezil with first-order absorption well. In EMs, trough concentrations were reported to be approximately half the plasma concentration at $2 \mathrm{~h}$ after oral administration, according to the pharmacokinetic parameters [32]. Hence, the plasma donepezil concentrations in patients of this study might have been below the upper level of the therapeutic range. We did not find significant differences between patients with NPI scores above or below 20 points, possibly because of the small number (four) of patients who developed severe BPSD.

CYP2D6 is a major enzymatic pathway for the degradation of donepezil, and CYP3A4/5 is the alternative pathway in men [18]. No significant gene or phenotype effects of CYP2D6 and CYP $3 A 5^{*} 3$ on the development of $B P S D$ were seen. Based on the speculation that high plasma donepezil concentrations might improve BPSD, intermediate metabolizers of CYP2D6 should have shown lower NPI scores than those of extensive metabolizers, or ultrarapid metabolizers. However, no phenotype-depen- dent improvement in BPSD was seen. This result was supported by the finding that plasma donepezil concentrations did not increase in a phenotype-dependent manner [22]. Since all the patients with high donepezil doses $(>0.20 \mathrm{mg} / \mathrm{kg})$ showed NPI scores below 20 points, a combined analysis of phenotypes and doses may be needed to clarify the influence of CYP2D6 phenotypes on the development of BPSD with a larger patient cohort.

$A B C B 1$ gene encodes $\mathrm{P}$-glycoprotein, and decreased activity of $\mathrm{P}$-glycoprotein due to genetic variants might cause a high donepezil concentration in the brain. We did not find a significant influence of $A B C B 1$ genotypes on NPI scores. Although many studies have investigated the influence of the ABCB1 G2677T/A and the C3435T genotype or haplotype on the activity of P-glycoprotein, the significance of the variant is still unclear [33-36]. Since donepezil is a substrate of the breast cancer resistance protein [37], further studies may be needed to clarify the influence of polymorphisms of the breast cancer resistance protein on the development of BPSD.

Yokukansan is a Japanese Kampo medicine that consists of crude extracts from Atractylodes lancea rhizome, 
Poria sclerotium, Cnidium rhizome, Uncaria hook, Japanese Angelica root, Bupleurum root, and Glycyrrhiza. Yokukansan is sometimes prescribed for BPSD to Alzheimer's patients in Japan [38]. NPI scores in patients receiving Yokukansan, psychotropics, or memantine did not differ from those not receiving the drugs. Moreover, there was no significant difference in plasma donepezil concentrations between patients receiving and not receiving Yokukansan [22]. These results indicate that the effect of Yokukansan on BPSD is independent from that of donepezil.

This study had several limitations. The average blood sampling time was $2.1 \mathrm{~h}$ after the last administration of donepezil. We did not periodically conduct MMSE because the primary goal of this study was to investigate the relationship between the plasma donepezil concentration and development of BPSD, not cognitive function.

\section{Conclusions}

Although we did not find a direct relationship between plasma donepezil pharmacokinetics and NPI scores in Alzheimer's patients, higher plasma donepezil concentrations might be a promising factor in preventing or alleviating severe BPSD.

\section{Statement of Ethics}

The research was conducted ethically in accordance with the World Medical Association Declaration of Helsinki, Ethical Guidelines for Medical Research for Humans from the Japanese Ministry of Health, Labor and Welfare, and the Rules of the Ethics
Committee of the National Epilepsy Center, Shizuoka Institute of Epilepsy and Neurological Disorders and the University of Shizuoka. This study was approved by the Institutional Review Boards at the National Epilepsy Center, Shizuoka Institute of Epilepsy and Neurological Disorders (No. 2013-33), and the University of Shizuoka (No. 27-3). All patients or their families provided written informed consent to a Statement of Ethics.

\section{Conflict of Interest Statement}

The authors declare that there are no competing interests for this work.

\section{Funding Sources}

This study was supported by JSPS KAKENHI Grant Number JP25460215.

\section{Author Contributions}

Dr. Kagawa designed and supervised the study and wrote the manuscript. Dr. Yamamoto gathered informed consent from the patients. Dr. Kagawa, Ms. Ueno, Mr. Inomata, and Ms. Tezuka measured drug concentrations and performed gene analysis. Drs. Osawa and Yazawa designed the study and edited the manuscript. Dr. Maeda performed the gene analysis. Dr. Obi recruited patients and provided professional medical advice.

\section{Data Availability Statement}

The data that support the findings of this study are available from the corresponding author, Y.K., upon reasonable request.

\section{References}

1 Lane CA, Hardy J, Schott JM. Alzheimer's disease. Eur J Neurol. 2018;25(1):59-70.

2 Feast A, Moniz-Cook E, Stoner C, Charlesworth $\mathrm{G}$, Orrell M. A systematic review of the relationship between behavioral and psychological symptoms (BPSD) and caregiver wellbeing. Int Psychogeriatr. 2016;28(11):176174.

3 Spalletta G, De Luca V, Padovani A, Rozzini L, Perri R, Bruni A, et al. Early onset versus late onset in Alzheimer's disease: what is the reliable cut-off? Adv Alzheimer Dis. 2013; 2(1):40-7.

4 Fernández M, Gobartt AL, Balañá M; COOPERA Study Group. Behavioural symptoms in patients with Alzheimer's disease and their association with cognitive impairment. BMC Neurol. 2010;10:87.
5 Wang F, Feng TY, Yang S, Preter M, Zhou JN, Wang XP. Drug therapy for behavioral and psychological symptoms of dementia. Curr Neuropharmacol. 2016;14(4):307-13.

6 Okahara K, Ishida Y, Hayashi Y, Inoue T, Tsuruta K, Takeuchi K, et al. Effects of Yokukansan on behavioral and psychological symptoms of dementia in regular treatment for Alzheimer's disease. Prog Neuropsychopharmacol Biol Psychiatry. 2010;34(3):532-6.

7 Matsunaga S, Kishi T, Iwata N. Yokukansan in the treatment of behavioral and psychological symptoms of dementia: an updated metaanalysis of randomized controlled trials. J Alzheimers Dis. 2016;54(2):635-43.

8 Takeyoshi K, Kurita M, Nishino S, Teranishi M, Numata Y, Sato T, et al. Yokukansan improves behavioral and psychological symp- toms of dementia by suppressing dopaminergic function. Neuropsychiatr Dis Treat. 2016; 12:641-9.

9 Tible OP, Riese F, Savaskan E, von Gunten A. Best practice in the management of behavioural and psychological symptoms of dementia. Ther Adv Neurol Disord. 2017;10(8): 297-309.

10 Barak Y, Bodner E, Zemishlani H, Mirecki I, Aizenberg D. Donepezil for the treatment of behavioral disturbances in Alzheimer's disease: a 6-month open trial. Arch Gerontol Geriatr. 2001;33(3):237-41.

11 Paleacu D, Mazeh D, Mirecki I, Even M, Barak Y. Donepezil for the treatment of behavioral symptoms in patients with Alzheimer's disease. Clin Neuropharmacol. 2002; 25(6):313-7. 
12 Lockhart IA, Orme ME, Mitchell SA. The efficacy of licensed-indication use of donepezil and memantine monotherapies for treating behavioural and psychological symptoms of dementia in patients with Alzheimer's disease: systematic review and meta-analysis. Dement Geriatr Cogn Dis Extra. 2011;1(1): 212-27.

13 Campbell N, Ayub A, Boustani MA, Fox C, Farlow M, Maidment I, et al. Impact of cholinesterase inhibitors on behavioral and psychological symptoms of Alzheimer's disease: a meta-analysis. Clin Interv Aging. 2008;3(4): 719-28.

14 Kimura T, Takamatsu J. Two cases of Alzheimer's disease showing deterioration of behavioral and psychological symptoms of dementia induced by switching from rivastigmine to donepezil. Neuropsychiatr Dis Treat. 2013;9:49-53.

15 Sasaki S, Horie Y. The effects of an uninterrupted switch from donepezil to galantamine without dose titration on behavioral and psychological symptoms of dementia in Alzheimer's disease. Dement Geriatr Cogn Dis Extra. 2014;4(2):131-9.

16 Suzuki H, Inoue Y, Mikami K, Gen K. The influence and changes in the dosages of concomitantly used psychotropic drugs associated with the discontinuation of donepezil in severe Alzheimer's disease with behavioral and psychological symptoms on dementia: a preliminary open-label trial. Ther Adv Psychopharmacol. 2014;4(1):37-42.

17 Barner EL, Gray SL. Donepezil use in Alzheimer disease. Ann Pharmacother. 1998;32(1): 70-7.

18 Coin A, Pamio MV, Alexopoulos C, Granziera S, Groppa F, de Rosa G, et al. Donepezil plasma concentrations, CYP2D6 and CYP3A4 phenotypes, and cognitive outcome in Alzheimer's disease. Eur J Clin Pharmacol. 2016;72(6):711-7.

19 Matsui K, Mishima M, Nagai Y, Yuzuriha T, Yoshimura T. Absorption, distribution, metabolism, and excretion of donepezil (Aricept) after a single oral administration to rat. Drug Metab Dispos. 1999;27(12):1406-14.

20 Magliulo L, Dahl ML, Lombardi G, Fallarini S, Villa LM, Biolcati A, et al. Do CYP3A and
$\mathrm{ABCB} 1$ genotypes influence the plasma concentration and clinical outcome of donepezil treatment? Eur J Clin Pharmacol. 2011;67(1): 47-54.

21 Zhong Y, Zheng X, Miao Y, Wan L, Yan H, Wang B. Effect of CYP2D6*10 and APOE polymorphisms on the efficacy of donepezil in patients with Alzheimer's disease. Am J Med Sci. 2013;345(3):222-6.

22 Kagawa Y, Yamamoto Y, Ueno A, Maeda T, Obi T. Impact of polymorphisms of CYP2D6, $\mathrm{CYP} 3 \mathrm{~A} 5$, and $\mathrm{ABCB} 1$ on plasma concentrations of donepezil and its metabolite in patients with Alzheimer's disease. Ther Drug Monit. 2021 Jun 1;43(3):429-35.

23 Ishiwata K, Kawamura K, Yanai K, Hendrikse $\mathrm{NH}$. In vivo evaluation of P-glycoprotein modulation of 8 PET radioligands used clinically. J Nucl Med. 2007;48(1):81-7.

24 Balram C, Zhou Q, Cheung YB, Lee EJ. CY$\mathrm{P}^{\mathrm{A} 5 * 3}$ and $* 6$ single nucleotide polymorphisms in three distinct Asian populations. Eur J Clin Pharmacol. 2003;59(2):123-6.

25 Tanabe M, Ieiri I, Nagata N, Inoue K, Ito S, Kanamori Y, et al. Expression of P-glycoprotein in human placenta: relation to genetic polymorphism of the multidrug resistance (MDR)-1 gene. J Pharmacol Exp Ther. 2001; 297(3):1137-43.

26 Cummings JL. The neuropsychiatric inventory: assessing psychopathology in dementia patients. Neurology. 1997;48(5 Suppl 6):S10 6.

27 Clerici F, Vanacore N, Elia A, Spila-Alegiani S, Pomati S, Da Cas R, et al. Memantine effects on behaviour in moderately severe to severe Alzheimer's disease: a post-marketing surveillance study. Neurol Sci. 2012;33(1):23-31.

28 Folstein MF, Folstein SE, McHugh PR. Cognitive defect in medical illness. Ann Intern Med. 1977;86(6):827-8.

29 Pilli NR, Inamadugu JK, Kondreddy N, Karra VK, Damaramadugu R, Rao JV. A rapid and sensitive LC-MS/MS method for quantification of donepezil and its active metabolite, 6-o-desmethyl donepezil in human plasma and its pharmacokinetic application. Biomed Chromatogr. 2011;25(8):943-51.

30 Shah HJ, Kundlik ML, Pandya A, Prajapati S, Subbaiah G, Patel CN, et al. A rapid and spe- cific approach for direct measurement of donepezil concentration in human plasma by LC-MS/MS employing solid-phase extraction. Biomed Chromatogr. 2009;23(2):14151.

31 Hiemke C, Bergemann N, Clement HW, Conca A, Deckert J, Domschke K, et al. Consensus guidelines for therapeutic drug monitoring in neuropsychopharmacology: update 2017. Pharmacopsychiatry. 2018;51(1-02): e1-62.

32 Noetzli M, Guidi M, Ebbing K, Eyer S, Wilhelm L, Michon A, et al. Population pharmacokinetic approach to evaluate the effect of CYP2D6, CYP3A, ABCB1, POR and NR1I2 genotypes on donepezil clearance. Br J Clin Pharmacol. 2014;78(1):135-44.

33 Takatori R, Takahashi KA, Tokunaga D, Hojo T, Fujioka M, Asano T, et al. ABCB1 C3435T polymorphism influences methotrexate sensitivity in rheumatoid arthritis patients. Clin Exp Rheumatol. 2006;24(5):546-54.

34 Kim RB, Leake BF, Choo EF, Dresser GK, Kubba SV, Schwarz UI, et al. Identification of functionally variant MDR1 alleles among European Americans and African Americans. Clin Pharmacol Ther. 2001;70(2):189-99.

35 Ishikawa M, Kawai M, Maeda T, Kagawa Y. Prediction of neutrophil reduction using plasma paclitaxel concentration after administration in patients with uterine, ovarian, or cervical cancers in an outpatient clinic. Cancer Chemother Pharmacol. 2018;81(2):399-411.

36 Yaowaluk T, Senanarong V, Limwongse C, Boonprasert R, Kijsanayotin P. Influence of CYP2D6, CYP3A5, ABCB1, APOE polymorphisms and nongenetic factors on donepezil treatment in patients with Alzheimer's disease and vascular dementia. Pharmgenomics Pers Med. 2019;12:209-24.

37 Takeuchi R, Shinozaki K, Nakanishi T, Tamai I. Local drug-drug interaction of donepezil with cilostazol at breast cancer resistance protein (ABCG2) increases drug accumulation in heart. Drug Metab Dispos. 2016;44(1):68-74.

38 Fukui M, Katayama S, Ikeya Y, Inazu T. Yokukansan, a Kampo medicine, enhances the level of neuronal lineage markers in differentiated P19 embryonic carcinoma cells. Heliyon. 2019;5(10):e02662. 